\title{
Large scale magnetic fields and their dissipation in GRB fireballs
}

\author{
H. C. Spruit, F. Daigne, and G. Drenkhahn \\ Max-Planck-Institut für Astrophysik, Postfach 1317, 85741 Garching bei München, Germany
}

Received 21 April 2000 / Accepted 17 January 2001

\begin{abstract}
We consider possible geometries of magnetic fields in GRB outflows, and their evolution with distance from the source. For magnetically driven outflows, with an assumed ratio of magnetic to kinetic energy density of order unity, the field strengths are sufficient for efficient production of $\gamma$-rays by synchrotron emission in the standard internal shock scenario, without the need for local generation of small scale fields. In these conditions, the MHD approximation is valid to large distances $\left(\gtrsim 10^{19} \mathrm{~cm}\right)$. In outflows driven by nonaxisymmetric magnetic fields, changes of direction of the field cause dissipation of magnetic energy by reconnection. Much of this dissipation takes place outside the photosphere of the outflow, and can convert a significant fraction of the magnetic energy flux into radiation.
\end{abstract}

Key words. gamma-ray bursts - magnetic fields - radiation mechanisms: nonthermal - shock waves

\section{Introduction}

Several models for cosmic $\gamma$-ray bursts (hereafter GRBs) make use of rapidly rotating compact stellar-mass sources. Though many details in each case are uncertain, the two more developed and popular scenarios involve the coalescence of two compact objects (neutron star + neutron star or neutron star + black hole) and the collapse of a massive star to a black hole (collapsar) (Mészáros \& Rees 1992; Narayan et al. 1992; Woosley 1993; Paczyński 1998). Both lead to the same system: a stellar mass black hole surrounded by a thick torus made of stellar debris or of infalling stellar material partially supported by centrifugal forces. An other interesting proposition (Usov 1992; Kluzniak \& Ruderman 1998; Spruit 1999) associates GRBs with highly magnetized millisecond pulsars.

The energy release by such a source can be fed from various reservoirs. In the case of a thick disk + black hole system, the burst can be powered by the accretion of disk material by the black hole or by extracting directly the rotational energy of the black hole via the Blandford-Znajek mechanism. In the case of a highly magnetised millisecond pulsar the energy release comes from the rotational energy of the neutron star.

Luminosities as high as those of GRBs cannot be radiated in the close vicinity of the source. The energy released must first drive a wind which rapidly becomes relativistic. Its kinetic energy is then converted into $\gamma$-rays (producing the prompt emission of the GRB) at large radii via the formation of shocks, probably within the wind itself

Send offprint requests to: H. C. Spruit, e-mail: henk@mpa-garching.mpg.de (internal shock model) (Rees \& Mészáros 1994; Daigne \& Mochkovitch 1998). The wind is finally decelerated by the external medium, which leads to a new shock responsible for the afterglow emission observed in X-rays, optical and radio bands (external shock model) (Katz 1994; Rhoads \& Paczynski 1993; Sari \& Piran 1997; Wijers et al. 1997).

The Lorentz factor $\Gamma$ of the relativistic wind must reach high values $\left(\Gamma \sim 10^{2}-10^{3}\right)$ both to produce $\gamma$-rays and to avoid photon-photon annihilation along the line of sight, whose signature is not observed in the spectra of GRBs (Goodman 1986, see also Baring 1995). This limits the allowed amount of baryonic pollution in the flow to a very low level and makes the production of the relativistic wind a challenging problem. Only a few ideas have been proposed and none appears to be fully conclusive at present.

However it is suspected that large magnetic fields play an important role. This is obvious in models using a highly magnetised millisecond pulsar, but in the case of a thick disk + black hole system a magnetic wind is also probably more efficient than the initial proposition where the wind is powered by the annihilation of neutrino-antineutrinos pairs along the rotational axis (Berezinskii \& Prilutskii 1987; Goodman et al. 1987; Ruffert et al. 1997). The magnetic field in the disk could be amplified by differential rotation to very large values $\left(B \sim 10^{15} \mathrm{G}\right)$ and a magnetically driven wind could then be emitted from the disk (Thompson 1994; Mészáros \& Rees 1997). under severe constraints on the field geometry and the dissipation close to the disk, large values of the lorentz factor can then be reached (Daigne \& Mochkovitch 2000c, 2000a).

The emission of photons at large radii via the formation of shocks is perhaps better understood than the 
central engine. It is believed that a non-thermal population of accelerated electrons is produced behind the shock waves and synchrotron and/or inverse Compton photons are emitted. A magnetic field is then required. In the case of the afterglow emission, the external shock is propagating in the environment of the source, and the magnetic field has to be locally generated by microscopic processes (Mészáros \& Rees 1993; Wijers 1997; Thompson \& Madau 2000). In the case of the prompt $\gamma$-ray emission which is probably due to internal shocks within the wind, such a locally generated magnetic field is also usually invoked (Rees \& Mészáros 1994; Papathanassiou \& Mészáros 1996; Sari \& Piran 1997; Daigne \& Mochkovitch 1998). It has also been pointed out that a large scale field originating from the source could play the same role (Mészáros \& Rees 1993, 1997; Tavani 1996).

The argumentation in this paper is organized as follows. In Sect. 2 we show that for typical baryon loading, the particle density in the outflow is so large that the MHD approximation is appropriate out to distances of the order $10^{20} \mathrm{~cm}$. This will turn out to be larger than the other relevant distances.

Due to the baryon loading, the GRB case is therefore different from the essentially baryon-free pulsar wind case (e.g. Usov 1994), where the MHD approximation breaks down much earlier, and plasma theories of large amplitude electromagnetic waves (LAEMW) are applied. The GRB case is actually simpler than the pulsar case in this respect.

The evolution of the magnetic field can therefore be dealt with in MHD approximation. In Sect. 3 we discuss how the strength and configuration of the field in the outflow depends on assumptions about the central engine. This is done first without allowing for decay of the field by internal MHD processes. In Sect. 4 we show that the field strengths so obtained are sufficient to produce synchrotron and/or inverse Compton emission in the standard internal shock model, without the need for local generation of microscopic magnetic fields. In Sect. 5 we then argue that internal MHD processes are, in fact, likely to cause magnetic field energy density to be released during the outflow. This may be a significant contributor to the observed emission. The efficiency of conversion of the primary energy of the central engine to observable $\gamma$-rays can also be much higher than in internal shock models. The arguments are summarized again in Sect. 7 .

\section{The validity of the MHD approximation}

In this section, as well as throughout this paper, a prime denotes quantities measured in the rest frame of the outflowing matter, unprimed quantities are measured in the "laboratory" frame (understood here as a frame at rest relative to the central object of the burst).

In order to maintain the necessary current there must be enough plasma available. As an example, consider the case of a magnetized outflow with magnetic field of alternating direction, as happens in a pulsar-like model (Sect. 3.4). We approximate this as a plane sinusoidal wave with a wave number $k$ and a angular frequency $\Omega$ :

$$
\begin{aligned}
& \boldsymbol{E}=E \sin (k z-\Omega t) \boldsymbol{e}_{x} \\
& \boldsymbol{B}=B \sin (k z-\Omega t) \boldsymbol{e}_{y}
\end{aligned}
$$

In a frame comoving with the fluid the field strength is time-independent, hence the phase speed of the wave in the lab frame is $\Omega / k=\beta c$. From the induction equation $\nabla \times \boldsymbol{E}=-\partial_{\mathrm{t}} \boldsymbol{B} / c$ the relation between the electrical and magnetic field amplitudes is $E=\beta B$. Ampère's equation gives the current density, $\boldsymbol{j}=-\Omega /(4 \pi) B\left(\beta^{-1}-\beta\right) \cos (k z-$ $\Omega t) \boldsymbol{e}_{x}$, or, with $\Gamma=\left(1-\beta^{2}\right)^{-1 / 2}$ :

$j=\frac{c k B}{4 \pi \Gamma^{2}}$

(The same result is obtained if the current density is calculated in MHD approximation in the comoving frame and then Lorentz transformed back into the lab frame.) The current that can be maintained by the outflowing medium is limited by the density of charged particles $n$. The maximum current density (in the comoving frame) is $j_{\mathrm{m}}^{\prime}=n^{\prime} e c$, when the particles are given their maximum speed $c$. In the lab frame this current density is (Melatos \& Melrose 1996)

$j_{\mathrm{m}}=n e c / \Gamma$.

If $j$ gets larger than $j_{\mathrm{m}}$ the simple ansatz of an electromagnetic wave travelling with the same speed as the plasma cannot hold any longer. Thus flux freezing is impossible and MHD is not sufficient any more to describe the problem.

Plasma physical instabilities can set in at current densities much lower than (4). They will produce an anomalous resistivity in the plasma so that an electric field is present also in the rest frame of the plasma. The electric field due to this resistivity is small, however, compared to the magnetic field strength as long as the charged particle density is larger than the minimum density. The MHD approximation in this sense is then a good one on large scales, in spite of the presence of small scale plasma processes.

If the outflow has a finite duration $\tau$, and a constant speed $\beta c$ in this time interval, it moves as a shell of width $\beta c \tau \approx c \tau$. When the shell (assumed spherical) has expanded to a radius $r$, the particle density measured in the lab frame is $n(r)=M /\left(2 \pi r^{2} c \tau m_{\mathrm{p}}\right)$ where $M$ is the total mass ejected and $m_{\mathrm{p}}$ the proton mass. With (3) and (4), the minimum particle density $n_{\mathrm{c}}$ is

$n_{\mathrm{c}}(r)=\frac{k B}{4 \pi e \Gamma}$

If internal MHD processes can be neglected, the total electro-magnetic energy $E_{\mathrm{em}}$ of the shell is conserved (see Sect. 3). The field strength in the lab frame is then

$B=\sqrt{\frac{2 E_{\mathrm{em}}}{c \tau r^{2}}}$. 
With (5), and the known density $n(r)$, we thus find that the MHD approximation breaks down when the shell has reached the critical radius

$r_{\mathrm{c}}=\frac{e E_{\mathrm{k}}}{\Omega m_{\mathrm{p}} c} \sqrt{\frac{2}{E_{\mathrm{em}} \tau c}}=\frac{e}{\Omega m_{\mathrm{p}} c} \sqrt{\frac{2}{\xi(\xi+1)} \frac{E}{c \tau}}$

where $E_{\mathrm{k}}=\Gamma M c^{2}$ is the kinetic energy, $\xi=E_{\mathrm{em}} / E_{\mathrm{k}}$ (in the lab frame) and $E=E_{\mathrm{em}}+E_{\mathrm{k}}$ is the total energy. Assuming $\xi$ to be of the order unity we have

$r_{\mathrm{c}} \approx \frac{e}{\Omega m_{\mathrm{p}} c} \sqrt{\frac{E}{c \tau}}=3.210^{20} \mathrm{~cm} \cdot E_{52}^{1 / 2} \Omega_{4}^{-1}\left(\frac{\tau}{3 \mathrm{~s}}\right)^{-1}$.

The processes discussed in this paper happen well within time scales smaller than $r_{\mathrm{cr}} / c$, and the MHD is justified.

In baryon-free cases, the expected values of $\xi$ may be much higher (Usov 1994), as in the typical pulsar-wind scenarios (Kennel \& Coroniti 1984; Begelman \& Li 1992; Blackman \& Yi 1998), and the critical radius for MHD condition correspondingly smaller. Such low baryon loading seems rather unlikely, however, for the currently proposed scenarios for GRB engines. Outflows from accretion disks, merging stars, and supernova envelopes are all intrinsically highly baryon loaded environments, and have some difficulties reaching baryon loadings as small as $\eta \sim 10^{-3}-10^{-4}$. Even in the rapidly magnetized msec neutron star scenario (Spruit 1999), emergence of magnetic fields from the star at the required short time scales is likely to imply that some baryonic mass is lifted together with the magnetic fields.

\section{Field geometries}

A burst of duration $\tau$ produces a shell of width $d=c \tau$, travelling outward at a speed $\beta c$. The observed radiation from this shell is produced when it has expanded to a radius $r \gg d$. This thin shell carries with it magnetic field lines from the source, which we call "trapped field lines". For the rest of this section, we assume a relativistic outflow, $\beta \approx 1$.

\subsection{Trapped fields}

If reconnection processes inside the shell can be neglected, the field lines are frozen in the expanding shell. If $\boldsymbol{B}=$ $\left(B_{\mathrm{r}}, B_{\theta}, B_{\phi}\right)$ (in spherical coordinates), and the width of the shell is constant, the components then vary with distance as $B_{\mathrm{r}} \sim r^{-2}, B_{\theta} \sim B_{\phi} \sim r^{-1}$. This is because the radial component is divided over the surface of the spherical shell, while the components parallel to the shell surface decrease with the circumference. More formally, the induction equation $\partial_{\mathrm{t}} \boldsymbol{B}=\nabla \times(\boldsymbol{u} \times \boldsymbol{B})$ has the components

$$
\begin{aligned}
\partial_{\mathrm{t}} B_{\theta}= & (r \sin \theta)^{-1} \partial_{\phi}\left(u_{\theta} B_{\phi}-u_{\phi} B_{\theta}\right) \\
& -r^{-1} \partial_{\mathrm{r}}\left(r\left(u_{\mathrm{r}} B_{\theta}-u_{\theta} B_{\mathrm{r}}\right)\right) \\
\partial_{\mathrm{t}} B_{\phi}= & r^{-1}\left(\partial_{\mathrm{r}}\left(r\left(u_{\phi} B_{\mathrm{r}}-u_{\mathrm{r}} B_{\phi}\right)\right)\right. \\
& \left.-\partial_{\theta}\left(u_{\theta} B_{\phi}-u_{\phi} B_{\theta}\right)\right) .
\end{aligned}
$$
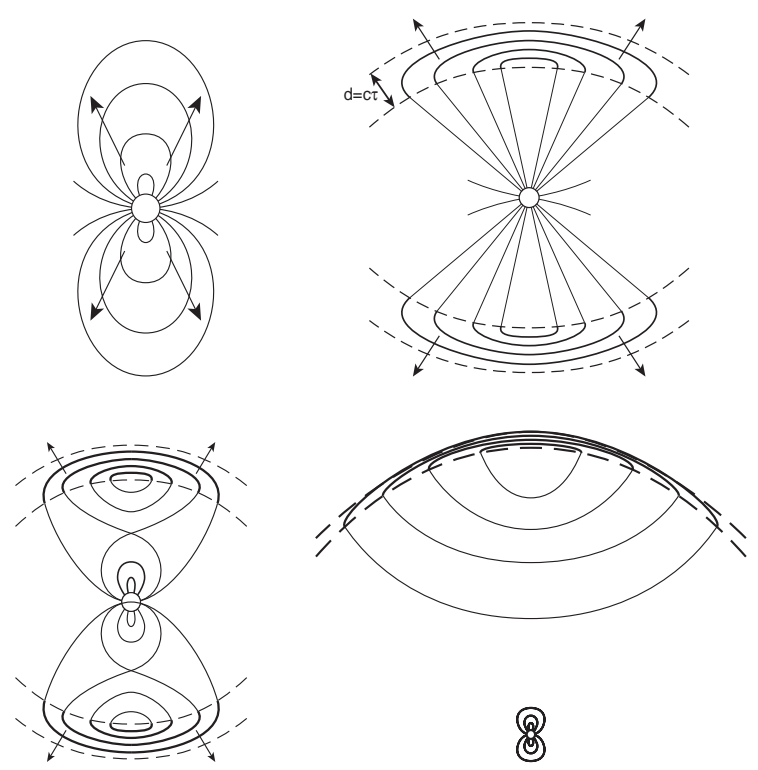

Fig. 1. Evolution of an initial dipole field by a radial outflow (schematic). Top left: initial field configuration. Top right: view on a larger scale, when the outflow, of finite duration $\tau$, now moves in a shell of width $c \tau$. It has stretched the field interior to the shell into a radial field. Lower left: reconnection in the low-density interior region restores the dipole field near the source. Lower right: as the shell moves out its thickness becomes small compared the distance travelled, and the further evolution depends on reconnection processes inside the shell

Assuming constant radial outflow $\left(u_{\mathrm{r}}=\right.$ const., $u_{\theta}=u_{\phi}=$ $0)$ and spherical symmetry $\left(\partial_{\theta}=\partial_{\phi}=0\right)$ the time evolution in a comoving fluid element is

$\frac{\mathrm{d} B_{\theta, \phi}}{\mathrm{d} t}=\partial_{\mathrm{t}} B_{\theta, \phi}+u_{\mathrm{r}} \partial_{\mathrm{r}} B_{\theta, \phi}=-r^{-1} \frac{\mathrm{d} r}{\mathrm{~d} t} B_{\theta, \phi}$.

Hence the tangential field $\boldsymbol{B}_{\mathrm{t}}=\left(B_{\theta}, B_{\phi}\right) \sim r^{-1}$. Since the expansion factor is very large between the source and the radius from which the emitted radiation reaches us, the radial component (varying as $\sim r^{-2}$ ) can be neglected and the field is almost exactly parallel to the surface of the shell (Fig. 1). If the width of the shell is constant, the magnetic energy in the shell $e_{\mathrm{m}}=\int B_{\mathrm{t}}^{2} \mathrm{~d} S$ is constant. The magnetic field thus carries a constant fraction of the kinetic energy of the outflow. Depending on how large this fraction is, the trapped field can be sufficient to produce the synchrotron emission proposed in internal shock models, without the need for "in situ" field generation mechanisms (Medvedev \& Loeb 1999).

How many field lines are trapped in the outflow, and hence which fraction of the outflow energy is magnetic, depends on conditions near the source. We consider here the representative possibilities.

\subsection{A "passive" magnetic field}

First consider a fireball expanding from the surface of a central object (not specified further) of radius $R$ and a dipolar magnetic field of moment $\mu$. We assume that the magnetic field plays no role in the driving of the outflow, 
but that it can confine plasma up to its own energy density $B^{2} / 8 \pi$.

Assume first that the expansion is kinematic, i.e. that the magnetic field is weak and its backreaction on the flow can be neglected. The number of field lines trapped in the outflow is equal to the flux of field lines outside crossing the dipole's equator outside the radius $R$ :

$\Phi=\frac{\pi \mu}{R}$.

This flux is a Lorentz invariant, so that the field strength of the shell (as measured in the frame of the central engine) is of the order $B=\Phi /(2 \pi r c \tau)$, or

$B \approx B_{0} \frac{R^{2}}{r c \tau}$

where $B_{0}=\mu / R^{3}$. Whether this is a large field strength, compared with the kinetic energy density $\Gamma \rho c^{2}$, depends on the assumed dipole moment $\mu$. An upper limit to the dipole moment follows from the requirement that the energy of the burst should be able to open the field lines of the central object. Let the energy of the burst be $\Gamma M c^{2}$, where $\Gamma$ the asymptotic Lorentz factor of the outflow and $M$ the baryon load. If this energy was initially confined in a region of size $R$ (the size of the central engine), the magnetic field strength $B_{0}$ in the confining dipole field of the source must satisfy
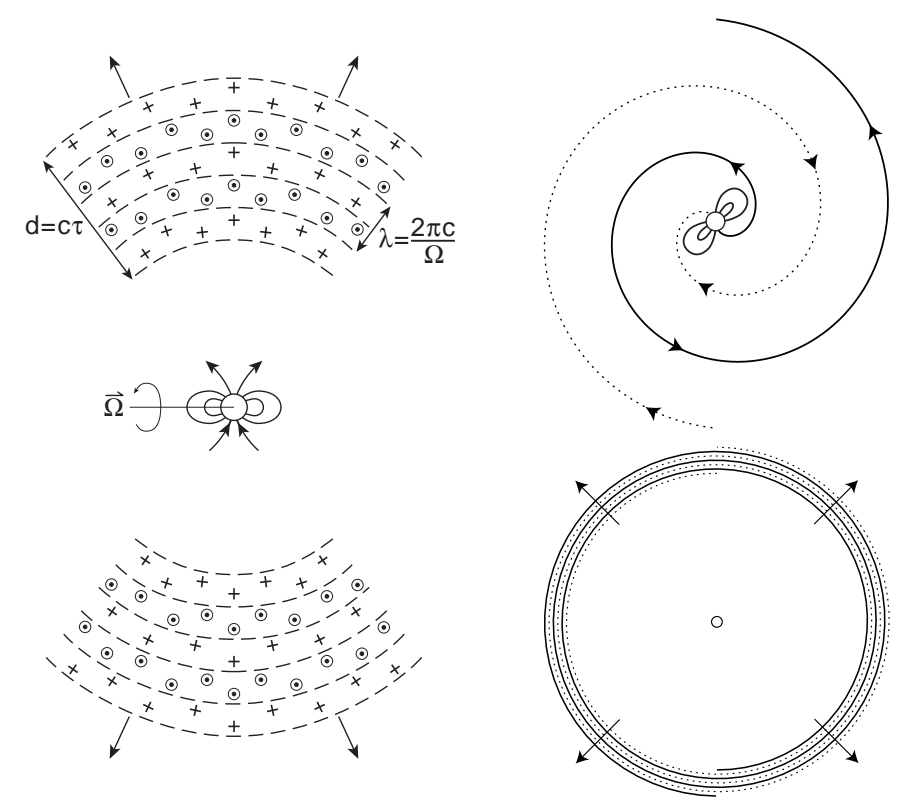

Fig. 2. Field configuration in quasi-spherical magnetic outflow driven by a perpendicular rotator ("pulsar-like" case) (schematic). Left: view in the equatorial plane, with dots and pluses indicating field lines into and out of the plane of the drawing. Right: top view from the rotational pole. Bottom right: same view on larger scale, at a later time $t \gg \tau$

$\frac{B_{0}^{2}}{8 \pi}<\frac{\Gamma M c^{2}}{\frac{4}{3} \pi R^{3}}$

in order for the field to be opened up by the fireball. With (13), the magnetic energy density at distance $r$ then satisfies

$\frac{B^{2}}{8 \pi}<e_{\mathrm{k}} \frac{R}{3 c \tau}$

where $e_{\mathrm{k}}$ is the kinetic energy density in the shell:

$e_{\mathrm{k}}=\frac{\Gamma M c^{2}}{4 \pi r^{2} c \tau}$.

For most central engine models considered, the duration of the burst is long compared to the light travel time across the source, $R / c \tau \ll 1$. A "passive" model, in which the magnetic field does not play a role in driving the outflow, therefore can only yield field strengths in the shell which are small compared with kinetic energy density. Even at such a low field strength, however, the magnetic field can become important for synchrotron emission in internal shocks, as discussed below in Sect. 4 .

\subsection{Active magnetic fields}

In magnetic models for GRB engines, the magnetic field serves to extract rotation energy from a rapidly rotating relativistic object. The details of such magnetic extraction (especially three-dimensional ones) are still somewhat uncertain, but basic energetic considerations are simple. Rotation of the mass-loaded field lines induces an azimuthal field component $B_{\phi}$. Let the distance from the

rotating object where this component is equal to the radial field be $r_{0}$. Except for cases with large baryon loading that are probably not relevant for GRB engines, $r_{0}$ is of the order of the Alfvén radius, which can lie anywhere between the surface of the object $R$ and the light cylin$\operatorname{der} r_{\mathrm{L}}=c / \Omega$. The energy output $L$ transmitted by the azimuthal magnetic stress $\left(B_{\phi} B_{\mathrm{r}} / 4 \pi\right)$ is then of the or$\operatorname{der} L \approx \Omega r_{0}^{3} B_{\mathrm{r}}^{2}\left(r_{0}\right)$. For a field dominated by its dipole component $\mu$, this yields a luminosity

$L=\Omega \mu^{2} / r_{0}^{3}$.

For $r_{0}=r_{\mathrm{L}}$, this yields (by order of magnitude) the pulsar spin-down formula for an inclined dipole rotating in vacuum, emitting an electromagnetic wave from its light cylinder.

The energy estimate (17) does not tell what the field configuration in the outflow looks like. The possibilities for dissipation of magnetic energy inside the outflow depend strongly on this configuration, which in turn depends on the nature of the magnetic field of the central engine. An important distinction is whether the rotating magnetic field is axisymmetric or nonaxisymmetric. In the nonaxisymmetric case, the outflow contains magnetic fields varying on the rather small scale $\pi c / \Omega$, the wavelength of the outgoing wave. In such a field, internal dissipation turns out to be much more likely to be important than in an axisymmetric field, where the length scale of the field is of the order of the distance $r$. In the following, this is illustrated with a few specific cases. 


\subsubsection{Ratio of magnetic to kinetic energy}

Estimate (17) only gives the total luminosity. Which fraction of it is in the form of kinetic energy and which in magnetic energy depends on more physics. At one extreme is Michel's (1969) model, in which the outflow consists of cold (pressureless) matter accelerated exclusively by the magnetic field. In this case, the ratio $\alpha=E_{\mathrm{m}} / E_{\mathrm{k}}$ of magnetic to kinetic luminosity is of the order $\xi \sim \Gamma^{2} \gg 1$. At large Lorentz factors, the energy is almost entirely in electromagnetic form, in this model. This is probably a property of the special geometry of the model, in which the flow is limited to the equatorial plane. If outflow in other directions is considered, much smaller values of $\xi$ result (Begelman \& Li 1994).

At the other extreme, consider a case in which most magnetic energy is released inside the light cylinder, in the form of a dense pair plasma (by some form of magnetic reconnection, for example). The result would then be just like the hydrodynamic expansion of a simple (Goodman 1986; Paczyński 1986) pair plasma fireball, and the resulting outflow would have $\xi \ll 1$. If some but not all energy is dissipated close to the source, intermediate values of $\xi$ would result. Since these important questions have not been resolved yet, we keep $\xi$ as a free parameter in the following. Where necessary we assume that it typically has values or order unity.

\subsection{Nonaxisymmetric quasi-spherical outflow}

Assume we have a perpendicular rotator, i.e. the rotating object has its dipole axis orthogonal to the rotation axis. At the source surface $r_{0}$, the rotating field is then strongest at the equator, and one expects the energy flux to be largest near the equatorial plane. With each rotation, a "stripe" consisting of a band of eastward and one of westward azimuthal field moves outward (Fig. 2, see also Coroniti 1990; Usov 1994). Assuming the outflow to be relativistic, the width $\lambda$ of such a stripe in the rest frame of the central object is $\lambda=2 \pi c / \Omega$. The (absolute value of the) azimuthal flux $\Phi$ in each half-wavelength is of the order of the poloidal flux outside the source surface $r_{0}, \Phi \approx 2 \pi \mu / r_{0}$. For spherical expansion of this amount of azimuthal flux, the field strength at distance $r$ is then

$B_{\phi} \approx \frac{\Phi}{r \lambda}=\frac{\mu}{r_{0} r_{\mathrm{L}} r}$

while the the total (magnetic plus kinetic) energy density $e_{\mathrm{k}}$ is

$e_{\mathrm{k}}+e_{\mathrm{m}}=\frac{L}{4 \pi r^{2} c}$

Hence with (17) the ratio of magnetic energy density to total energy density (in the lab frame) is of the order

$e_{\mathrm{m}} /\left(e_{\mathrm{k}}+e_{\mathrm{m}}\right) \approx \frac{r_{0}}{r_{\mathrm{L}}}$

\subsection{Jet}

Consider a collimated outflow along the axis of rotation (Fig. 3). This might be achieved by magnetic models in which the azimuthal field collimates the flow towards the axis by hoop stress (Bisnovatyi-Kogan \& Ruzmaikin 1976; Blandford \& Payne 1982; Sakurai 1985). In such a model, one assumes an axisymmetric (about the rotation axis) poloidal field, which is wound up into an azimuthal field wrapped around the axis. Let the opening angle of the outflow be $\theta$ (assumed constant), and $\varpi$ the cylindrical radius. At the source surface $\varpi_{0}=\theta z_{0}$ the poloidal and azimuthal field components are equal, and in the absence of magnetic reconnection processes

$$
\begin{aligned}
& B_{\phi}=B_{\mathrm{p} 0}\left(\varpi_{0} / \varpi\right)=B_{\mathrm{p} 0}\left(z_{0} / z\right), \\
& B_{\mathrm{p}}=B_{\mathrm{p} 0}\left(z_{0} / z\right)^{2} .
\end{aligned}
$$

If the collimation angle is small, the field as seen in a frame comoving with the jet is a slowly varying, nearly azimuthal field. Such a field is known to be highly unstable to kink instabilities (e.g. Bateman 1980). They operate on a time scale $\tau_{\mathrm{k}}$ of the order of the Alfvén crossing time, i.e. $\tau_{\mathrm{k}}=\varpi / v_{\mathrm{A}}$. Though the details of this process have not been worked out for jets (see however Lucek \& Bell $1996,1997)$, it is likely that the release of magnetic energy operates in two steps. In the first step, kink instability transforms the axisymmetric configuration into a nonaxisymmetric, helical, configuration. For an application to jets, see Königl \& Choudhuri (1985). This step is fast, operating on the Alvén crossing time. At this stage, the field has already lost much of its collimating ability, since the average azimuthal field strength has decreased in favour of a less ordered field component. During the instability, the so-called magnetic helicity is conserved, however, so that only a fraction of the magnetic energy is released. The further release of magnetic energy depends on reconnection processes. As discussed in Sect. 5, this is also likely to proceed on a time scale proportional to the Alfvén crossing time, though somewhat slower than the kink process itself.

As in the quasi-spherical outflow case, we ignore this internal dissipation of the magnetic energy for the moment, and return to it in Sect. 5. For a jet expanding with fixed opening angle $\theta$ (see Fig. 3), the field strength then varies as $r^{-1}$ and the magnetic energy is constant with distance. Since only a fraction of the magnetic energy is released in the kinking process, the ratio of magnetic to kinetic energy density is still of the order found in axisymmetric magnetic jet calculations (e.g. Camenzind 1987), i.e. of order unity:

$e_{\mathrm{m}} / e_{\mathrm{k}} \sim O(1)$.

\section{The emission expected from internal shocks}

\subsection{Typical parameters of an internal shock}

The internal shock model assumes that the initial distribution of the Lorentz factor in the shell is highly 

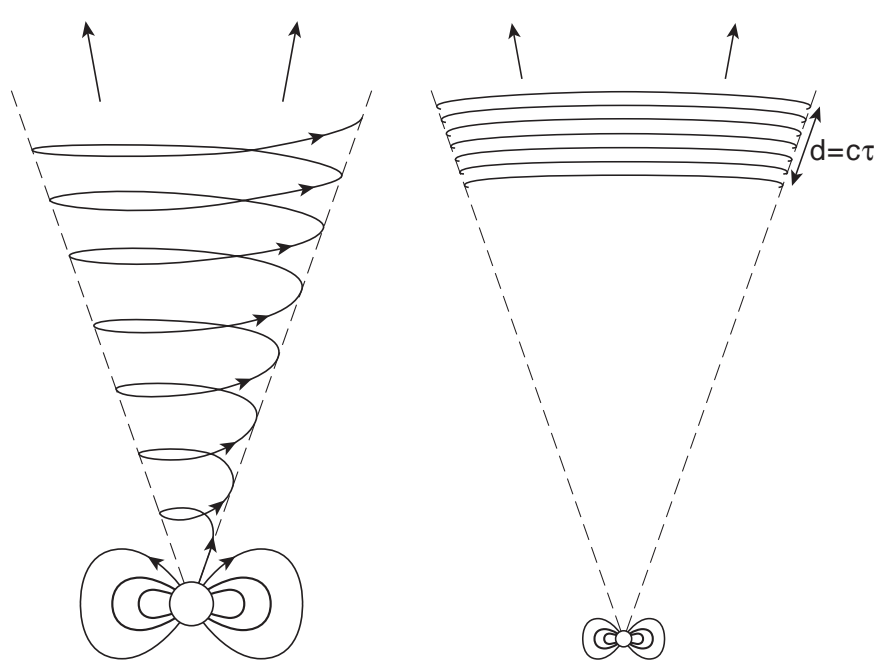

Fig. 3. Jet-like outflow of finite duration, magnetically driven by an axially symmetric rotator. Left: configuration near the source, showing how the field in the outflow is wound into a toroidal (azimuthal) field. Right: large scale view after a time long compared to the duration $\tau$. The field in the outflow is now a "pancake" of toroidal flux. Sketch ignores nonaxisymmetric processes like kink instability and subsequent reconnection, which can release energy from this configuration

variable. Rapid layers catch up with slower ones leading to internal shocks propagating within the relativistic shell. The hot material behind the shock waves is radiating efficiently and produces the observed prompt $\gamma$-ray emission of GRBs (Rees \& Mészáros 1994; Kobayashi et al. 1997; Daigne \& Mochkovitch 1998; Panaitescu \& Mészáros 1999; Daigne \& Mochkovitch 2000b). We only summarize here the basic assumptions of the model. For details see Daigne \& Mochkovitch (1998).

Consider two layers of equal mass (for simplicity) and of Lorentz factor $\Gamma_{1}$ and $\Gamma_{2}\left(\Gamma_{1}>\Gamma_{2}\right)$ which are emitted on a timescale $t_{\text {var }}$. They will collide at a radius

$$
\begin{aligned}
r_{\mathrm{IS}} & \simeq \frac{2 \Gamma_{1}^{2} \Gamma_{2}^{2}}{\Gamma_{1}^{2}-\Gamma_{2}^{2}} c t_{\mathrm{var}} \sim \Gamma^{2} c t_{\mathrm{var}} \\
& \sim 310^{15} \mathrm{~cm} \cdot\left(\frac{\Gamma}{300}\right)^{2}\left(\frac{t_{\mathrm{var}}}{1 \mathrm{~s}}\right)
\end{aligned}
$$

where $\Gamma$ is as usual the mean Lorentz factor of the flow. The average energy which is dissipated per proton in this shock is given by

$\epsilon=\left(\Gamma_{\text {int }}-1\right) m_{\mathrm{p}} c^{2}$

with

$\Gamma_{\text {int }}=\frac{1}{2}\left[\sqrt{\frac{\Gamma_{1}}{\Gamma_{2}}}+\sqrt{\frac{\Gamma_{2}}{\Gamma_{1}}}\right]$.

With $\Gamma_{1} / \Gamma_{2}=4$, which corresponds to a mildly relativistic shock $\left(v_{\text {rel }}=0.88 c\right)$, this gives $\epsilon \sim 240 \mathrm{MeV}$.

It is generally assumed (Rees \& Mészáros 1994; Papathanassiou \& Mészáros 1996; Sari \& Piran 1997; Daigne \& Mochkovitch 1998) that behind the shock wave a fraction of the electrons come into (at least partial) equipartition with the protons and become highly relativistic. If a fraction $\alpha_{\mathrm{e}}$ of the dissipated energy goes into a fraction $\zeta$ of the electrons, their characteristic Lorentz factor (in the comoving frame) will be

$\Gamma_{\mathrm{e}}^{\prime} \simeq \frac{\alpha_{\mathrm{e}}}{\zeta} \frac{\epsilon}{m_{\mathrm{e}} c^{2}}$

which, for complete equipartition and $\Gamma_{1} / \Gamma_{2} \sim 4$, yields $\Gamma_{\mathrm{e}}^{\prime} \sim 100-200$ if $\zeta \sim 1$ and $\Gamma_{\mathrm{e}}^{\prime} \gtrsim 10^{4}-210^{4}$ if only a small fraction of the electrons are accelerated $(\zeta \lesssim 0.01$. For some theoretical arguments in favour of such an assumption, see e.g. Bykov \& Mészáros 1996). Such highly relativistic electrons can emit $\gamma$-rays by the synchrotron and/or the inverse Compton process. A magnetic field is however required.

\subsection{Locally generated versus large-scale magnetic field}

The magnetic field involved in the synchrotron radiation is usually assumed to be generated locally by microscopic processes. Such a process has been proposed by Medvedev \& Loeb (1999). (This is also assumed for the external shock propagating in the interstellar medium and responsible for the afterglow; see however Thompson \& Madau 2000.) If this magnetic field is also into equipartition with the protons and electrons, it will have typical values of

$B_{\mathrm{eq}}^{\prime} \simeq \sqrt{8 \pi \alpha_{\mathrm{eq}} n^{\prime} \epsilon}$

where $\alpha_{\text {eq }} \leq 1 / 3$ and $n^{\prime}$ is the comoving proton number density, which can be estimated by

$$
\begin{aligned}
n^{\prime} & \simeq \frac{E_{\mathrm{k}}}{4 \pi r^{2} \Gamma^{2} c^{3} m_{\mathrm{p}} \tau} \\
& \simeq 2.010^{7} \mathrm{~cm}^{-3} \cdot r_{15}^{-2} E_{\mathrm{k}, 52}\left(\frac{\Gamma}{300}\right)^{-2} \tau_{1}^{-1} .
\end{aligned}
$$

For typical radii $r \sim 10^{15}-10^{16} \mathrm{~cm}$ and $\alpha_{\mathrm{eq}} \simeq \alpha_{\mathrm{e}} \simeq 1 / 3$, this leads to $B_{\text {eq }}^{\prime} \sim 100-1000 \mathrm{G}$ depending on the contrast $\Gamma_{1} / \Gamma_{2}$.

If the GRB is powered magnetically, however, the outflow is naturally magetized. (One good reason for assuming magnetic powering is that alternatives like $\nu \bar{\nu}$ annihilation are energetically inefficient.) As shown in Sect. 3, the magnetic energy content of the outflow is constant as long as internal dissipation of magnetic energy can be neglected. For the three cases considered, the ratio $\alpha_{\mathrm{LS}}$ of the (large scale-) magnetic to kinetic energy density is

$$
\begin{aligned}
\alpha_{\mathrm{LS}} & \equiv B^{2} /\left(8 \pi e_{\mathrm{K}}\right) \sim R /(c \tau), \\
\alpha_{\mathrm{LS}} & \sim r_{0} / r_{\mathrm{L}}, \\
\alpha_{\mathrm{LS}} & \sim O(1),
\end{aligned}
$$

for a passively expanded source field, a magnetically driven quasi-spherical outflow and a magnetically driven collimated jet respectively (cf. Eqs. (15, 20, 23)). For source sizes $R=10^{6}-10^{7} \mathrm{~cm}$ and durations $\tau=0.3-30 \mathrm{~s}$ the passively expanding field case has $\alpha_{\mathrm{LS}} \sim 10^{-6}-10^{-3}$. 
The magnetically driven quasi-spherical outflow would yield higher values, $\alpha_{\mathrm{LS}} \sim 0.1-1$, assuming $r_{0} / r_{\mathrm{L}} \sim$ 0.1-1. A magnetically driven jet would have a $\alpha_{\mathrm{LS}} \sim 1$. (These values hold as long as internal dissipation of magnetic energy can be ignored (Sect. 5)).

The corresponding comoving magnetic field in all cases is

$B_{\mathrm{LS}}^{\prime} \sim \frac{1}{\Gamma} \sqrt{8 \pi \alpha_{\mathrm{LS}} e_{K}} \sim \sqrt{8 \pi \alpha_{\mathrm{LS}} n^{\prime} m_{\mathrm{p}} c^{2}}$

which leads to (using the estimation of $n^{\prime}$ given by Eq. (31)):

$B_{\mathrm{LS}}^{\prime} \simeq 500 \mathrm{G} \cdot \alpha_{\mathrm{LS}}^{1 / 2} r_{15}^{-1} E_{\mathrm{k}, 52}^{1 / 2}\left(\frac{\Gamma}{300}\right)^{-1} \tau_{1}^{-1 / 2}$.

We see that

$\frac{B_{\mathrm{eq}}^{\prime}}{B_{\mathrm{LS}}^{\prime}} \simeq\left(\frac{\alpha_{\mathrm{eq}}}{\alpha_{\mathrm{LS}}}\right)^{1 / 2} \frac{\epsilon}{m_{\mathrm{p}} c^{2}}$

and that, depending on the values of $\alpha_{\text {eq }}$ and $\alpha_{\mathrm{LS}}$, the large scale magnetic field naturally has strengths comparable with the equipartition fields usually invoked.

\subsection{Synchrotron and inverse-compton emission}

Synchrotron emission by accelerated electrons in a magnetic field occurs at a typical energy (observer frame)

$E_{\text {syn }}=1.710^{-8} \mathrm{eV} \cdot \Gamma B^{\prime} \Gamma_{\mathrm{e}}^{\prime 2}$.

Consider first the case where a large fraction of the electrons takes part in the acceleration process, $\zeta \sim 1$. If the equipartition is complete - equal amounts of energy in protons and electrons (and magnetic fields if locally generated) - we then have $\Gamma_{\mathrm{e}}^{\prime} \sim 50-500$ so that for $B^{\prime} \sim 100$ $1000 \mathrm{G}$ the synchrotron photon energy

$E_{\text {syn }}=50 \mathrm{eV} \cdot\left(\frac{\Gamma}{300}\right) B_{3}^{\prime} \Gamma_{\mathrm{e}, 2}^{\prime 2}$

is usually in the $U V$ band (we don't consider here the high contrasts $\Gamma_{1} / \Gamma_{2} \simeq 1000$ which could produce synchrotron photons directly in the gamma-ray range even if $\zeta \sim 1$. Such high contrasts are probably not realistic in view of the difficulty to accelerate a wind to high Lorentz factors). $\gamma$-rays can then be produced by inverse Compton scattering on the synchrotron photons. We are in the Thomson limit where

$w=\frac{\Gamma_{\mathrm{e}}^{\prime} E_{\mathrm{syn}}^{\prime}}{m_{\mathrm{e}} c^{2}} \simeq 310^{-5} \cdot B_{3}^{\prime} \Gamma_{\mathrm{e}, 2}^{\prime 3} \ll 1$

so that

$$
\begin{aligned}
E_{\mathrm{IC}} & \simeq \Gamma_{\mathrm{e}}^{2} E_{\mathrm{syn}} \\
& \simeq 500 \mathrm{keV} \cdot\left(\frac{\Gamma}{300}\right) B_{3}^{\prime} \Gamma_{\mathrm{e}, 2}^{\prime 4} .
\end{aligned}
$$

The fraction of the electron energy which is radiated in the $\gamma$-ray range has been computed by Daigne \& Mochkovitch (1998) and is given by

$\alpha_{\mathrm{IC}} \simeq \frac{\tau_{*} \Gamma_{\mathrm{e}}^{\prime 2}}{1+\tau_{*} \Gamma_{\mathrm{e}}^{\prime 2}}$, where the optical $\tau_{*}$ depth of the layer of relativistic electrons is the solution of the equation

$$
\begin{aligned}
\tau_{*} \Gamma_{\mathrm{e}}^{\prime 2}\left(1+\tau_{*} \Gamma_{\mathrm{e}}^{\prime 2}\right) & \simeq 0.3 \cdot \frac{\alpha_{\mathrm{e}}}{\alpha_{\mathrm{eq}}} \\
& \simeq 0.06 \cdot\left(\frac{\epsilon}{200 \mathrm{MeV}}\right) \frac{\alpha_{\mathrm{e}}}{\alpha_{\mathrm{LS}}},
\end{aligned}
$$

for an equipartition field and a large-scale magnetic field respectively. Low values of the magnetic field increase the efficiency $\alpha_{\text {IC }}$ but $B^{\prime}$ cannot be too small because (i) $E_{\mathrm{IC}}$ must stay in the $\gamma$-ray range and (ii) the typical timescale of the radiative process $t_{\text {rad }}^{\prime}$ must be shorter than the expansion time $t_{\mathrm{ex}}^{\prime}$, otherwise the hot layer is cooling adiabatically. These two time-scales are

$t_{\mathrm{ex}}^{\prime} \simeq \frac{r}{\Gamma c} \simeq 100 \mathrm{~s} \cdot r_{15}\left(\frac{\Gamma}{300}\right)^{-1}$

and

$$
\begin{aligned}
t_{\mathrm{rad}}^{\prime} & \simeq \frac{t_{\mathrm{syn}}^{\prime}}{1+\tau_{*}{\Gamma_{\mathrm{e}}^{\prime}}^{2}} \\
& \simeq \frac{6 \mathrm{~s}}{1+\tau_{*} \Gamma_{\mathrm{e}}^{\prime 2}} B_{3}^{\prime-2} \Gamma_{\mathrm{e}, 2}^{\prime-1}
\end{aligned}
$$

The radiative efficiency (energy loss by radiation versus adiabatic cooling) is then of the order

$f_{\mathrm{rad}} \simeq \frac{t_{\mathrm{ex}}^{\prime}}{t_{\mathrm{ex}}^{\prime}+t_{\mathrm{rad}}^{\prime}}$

We see that (i) for the equipartition magnetic field these two conditions limit $\alpha_{\mathrm{eq}} / \alpha_{\mathrm{e}}$ to the range $0.01-1$ yielding low total efficiencies, $\alpha_{\text {IC }} \times f_{\text {rad }} \sim 0.4-0.1$; (ii) for a passively expanded source field $\left(\alpha_{\mathrm{LS}} \sim 10^{-6}-10^{-3}\right)$ it is impossible to produce $\gamma$-rays for the lowest values of $\alpha_{\mathrm{LS}}$ (both because $E_{\mathrm{IC}}$ is too low and $f_{\text {rad }}$ is very small). For $\alpha_{\mathrm{LS}} \sim 10^{-3}$ the magnetic field is still weak but for short bursts ( $n^{\prime}$ is higher) or high contrasts of Lorentz factors ( $\epsilon$ is higher) it is possible to produce a $\gamma$-ray burst with a total efficiency $\alpha_{\mathrm{IC}} \times f_{\text {rad }} \sim 0.6$ which is then quite high; (iii) for a magnetically driven quasi-spherical outflow $\left(\alpha_{\mathrm{LS}} \sim 0.1-1\right)$ the radiative efficiency is high $\left(f_{\mathrm{rad}} \sim 1\right)$, and there is no difficulty to produce $\gamma$-rays via the inverse Compton emission. However the efficiency of the IC process becomes very low if $\alpha_{\mathrm{LS}}$ is to close to unity $\left(\alpha_{\mathrm{IC}} \sim 0.02\right.$ for $\left.\alpha_{\mathrm{LS}}=1\right)$ and is still low $\left(\alpha_{\mathrm{IC}} \sim 0.2\right)$ for $\alpha_{\mathrm{LS}}=0.1$. Values of $\epsilon$ larger than $200 \mathrm{MeV}$ does not improve this efficiency much. (iv) For a magnetically driven jet the situation is very close to the previous case with $\alpha_{\mathrm{LS}} \sim 0.1$ and the IC efficiency is then very low.

We consider now the other extreme case where only a small fraction of the electrons are accelerated: $\zeta \lesssim 0.01$. The Lorentz factor of the electrons then reaches very high values of 5000-50000 and $\gamma$-rays can be produced directly by synchrotron emission:

$E_{\mathrm{syn}} \simeq 500 \mathrm{keV} \cdot\left(\frac{\Gamma}{300}\right) B_{3}^{\prime} \Gamma_{\mathrm{e}, 4}^{\prime 2}$ 
We are in the Klein-Nishina limit where

$w=\frac{\Gamma_{\mathrm{e}}^{\prime} E_{\mathrm{syn}}^{\prime}}{m_{\mathrm{e}} c^{2}} \simeq 30 \cdot B_{3}^{\prime} \Gamma_{\mathrm{e}, 4}^{\prime 3} \gg 1$.

The fraction of the energy which is directly radiated by synchrotron photons is now given by

$\alpha_{\mathrm{syn}} \simeq \frac{\tau_{*} \Gamma_{\mathrm{e}}^{2} / w}{1+\tau_{*} \Gamma_{\mathrm{e}}^{\prime 2} / w}$

and the optical depth is solution of a more complex equation (the opacity has decreased compared to the Thomson regime by a factor depending on $w)$ :

$$
\begin{aligned}
\frac{\tau_{*} \Gamma_{\mathrm{e}}^{\prime 2}}{w}\left(1+\frac{\tau_{*} \Gamma_{\mathrm{e}}^{2}}{w}\right) \simeq & 0.3 \cdot \frac{\alpha_{\mathrm{e}}}{\alpha_{\mathrm{eq}}} \frac{3}{8 w^{2}}(1+\ln (2 w)) \\
\simeq & 0.06\left(\frac{\epsilon}{200 \mathrm{MeV}}\right) \\
& \times \frac{\alpha_{\mathrm{e}}}{\alpha_{\mathrm{LS}}} \frac{3}{8 w^{2}}(1+\ln (2 w)),
\end{aligned}
$$

for the equipartition and large-scale magnetic field respectively. As before we also need to check that the radiative time-scale

$t_{\mathrm{rad}}^{\prime} \simeq \frac{0.06 \mathrm{~s}}{1+\tau_{*} \Gamma_{\mathrm{e}}^{\prime 2} / w} B_{3}^{\prime-2} \Gamma_{\mathrm{e}, 4}^{-1}$

is shorter than $t_{\text {ex }}^{\prime}$. This is now always the case $\left(f_{\mathrm{rad}} \sim 1\right)$ except for the lower values of $\alpha_{\mathrm{LS}}\left(10^{-6}\right)$ or for the last internal shocks occurring far from the source.

We then can conclude that (i) for an equipartition field $\alpha_{\text {eq }}$ has to be of the same order as $\alpha_{\mathrm{e}}$ in order for the synchrotron process is to produce $\gamma$-rays at high efficiency $\left(\alpha_{\text {syn }}>0.9\right)$. (ii) For a passively expanded source field the case $\alpha_{\mathrm{LS}} \sim 10^{-6}$ is again excluded because of an extremely low efficiency and a typical energy which is more in the $\mathrm{X}$-rays range. The case $\alpha_{\mathrm{LS}} \sim 10^{-3}$ suffers the same limitations as before, the efficiency is only $\alpha_{\text {syn }} \sim 0.1$. (iii) For a magnetically driven quasi-spherical outflow and a magnetically driven jet the situation is similar to the equipartition case: the efficiency is very high $\left(\alpha_{\text {syn }}>0.9\right.$ for a quasi-spherical outflow and $\alpha_{\text {syn }}>0.6$ for a jet).

In conclusion for this section, a passive expanded source field is probably too weak in most cases to produce a $\gamma$-ray burst without a locally generated magnetic field, but in the two other cases described in this paper, a magnetically driven quasi-spherical outflow and a magnetically driven collimated jet, there is no difficulty to produce $\gamma$-rays without any need of a supplementary field. The efficiencies of the radiative process in these magnetically driven cases are comparable to those already calculated for an equipartition field. In particular, as was already pointed out in Daigne \& Mochkovitch (1998), this efficiency is expected to be higher if the $\gamma$-rays are directly produced by synchrotron emission (which is possible if only a fraction of the electrons are accelerated behind the shock waves).

\section{Magnetic energy release in the outflow}

Internal release of magnetic energy can be important for the $\gamma$-ray lightcurves if the time scale is sufficiently fast that the release is significant before the outflow reaches the external shock. It should not be too fast, however. If the release takes place before the photospheric radius, i.e. in the optically thick part of the outflow, the internal energy generated is not radiated away. Instead, it is converted, through the radial expansion of the shell, into kinetic energy.

Assume that the outflow is driven by the rotating magnetic field of the central, compact object, i.e. that the field strength estimates (20) or (23) apply. Depending on the field configuration at the source, the field in the flow can be nonaxisymmetric to a greater or lesser degree, and depending on the nature of the acceleration process it can be quasi-spherical or more collimated along the rotation axis. Consider again the two cases discussed in Sect. 3 .

In the perpendicular rotator case, the field consists of "stripes" of alternating polarity, in which the field energy can be released by reconnection. In the axisymmetric jet case, the field is unstable to a kinking process. The magnetic field in both cases is far from a minimum energy configuration (a potential field). The free energy it contains is available if it can be released on a sufficiently short time scale.

The initial perturbations from which the MHD instabilities grow are likely to be present at significant amplitude, from the start of the outflow, except in the unlikely event that the field configuration of the source is highly symmetric. Thus we may assume that the ordered configurations of Figs. 2 and 3 significantly change to more disordered ones within an MHD instability time scale. These more disordered configurations then are subject to fast reconnection processes.

Reconnection takes place on time scales governed by the Alfvén speed. It depends on plasma resistivity as well, but in practical reconnection configurations (as opposed to highly symmetric textbook examples like tearing mode instability), the resistivity enters only weakly. In the Sweet-Parker model for 2-D reconnection, for example (e.g. Biskamp 1993), it enters through the logarithm of the magnetic Reynolds number. In more realistic 3-D modes of reconnection, the basic geometry of reconnection (a "chain link" kind of configuration) differs from the 2-D geometries. Also, the reconnection tends to be distributed over many current sheets instead of a few reconnection points (see Galsgaard \& Nordlund 1996, 1997, for recent numerical results). The reconnection rate is still weakly dependent on the resistivity in these $3-\mathrm{D}$ configurations.

\subsection{Perpendicular rotator}

In the perpendicular rotator, the field in the outflow changes (in the lab frame) on a length scale $L=\pi c / \Omega$. The time scale $\tau_{\mathrm{r}}$ of magnetic energy release scales with 
the Alfvén crossing time over this length scale. In a comoving frame

$1 / \tau_{\mathrm{r}}^{\prime} \approx \epsilon v_{\mathrm{A}}^{\prime} / L^{\prime}$

where $\epsilon<1$ is a numerical factor of order unity measuring the reconnection speed. In the lab frame, the energy release time scale is $\Gamma$ times longer, hence

$\tau_{\mathrm{r}}= \begin{cases}\frac{2 \pi \Gamma^{2} c}{\Omega \epsilon v_{\mathrm{A}}^{\prime}} & \text { (perpendicular rotator), } \\ \frac{\theta r \Gamma}{\epsilon v_{\mathrm{A}}^{\prime}} & \text { (axisymmetric jet). }\end{cases}$

As the bulk Lorentz factor $\Gamma$ tends to infinity, the energy release becomes arbitrarily slow. This is also understood by noting that for $\Gamma \rightarrow \infty$, the electromagnetic field of the outflow in the observer frame becomes indistinguishable from a pure EM wave in vacuum, whose energy content is conserved.

The relativistic Alfvén speed is (e.g. Jackson 1999)

$v_{\mathrm{A}}=\frac{c v_{\mathrm{B}}}{\left(c^{2}+v_{\mathrm{B}}^{2}\right)^{1 / 2}}$,

where $v_{\mathrm{B}}=B /(4 \pi \rho)^{1 / 2}$ is the nonrelativistic Alfvén speed.

Let the ratio of magnetic energy flux (Poynting flux) to kinetic energy flux be $\xi$ :

$\xi=\frac{B^{2}}{4 \pi \Gamma \rho c^{2}}=\frac{B^{\prime 2}}{4 \pi \rho^{\prime} c^{2}}$.

Hence

$v_{\mathrm{A}}^{\prime} / c=(1+1 / \xi)^{-1 / 2}$.

For the models discussed above, $\xi \sim O(1)$. As long as the magnetic energy density has not decayed by internal MHD processes, the comoving Alfvén speed is thus of the order of the speed of light.

The magnetic dissipation radius, the distance $r_{\mathrm{r}}$ from the source where magnetic energy release becomes important, is thus of the order

$$
\begin{aligned}
r_{\mathrm{r}} & =c \tau_{\mathrm{r}}=\frac{\pi c}{\epsilon \Omega} \Gamma^{2}(1+1 / \xi)^{1 / 2} \quad \text { (perp. rotator) } \\
& =210^{12} \mathrm{~cm} \cdot \Omega_{4}^{-1}\left(\frac{\Gamma}{300}\right)^{2} \frac{1}{\epsilon_{-1}}(1+1 / \xi)^{1 / 2}
\end{aligned}
$$

The length scale in the comoving frame, $L^{\prime}$, is equal to the wavelength $\lambda^{\prime}=2 \pi c \Gamma / \Omega=2 \pi r_{\mathrm{L}}$ in the perpendicular rotator case) or the lateral scale, $L^{\prime}=\theta r$ (in a collimated outflow).

\subsection{Axisymmetric jet}

For the case of an axisymmetric outflow along the rotation axis, the situation is a bit different. The length scale $L$ of the magnetic field is now the jet radius, $L \approx \theta r$, where $\theta$ is the opening angle of the jet. Since this is measured perpendicular to the flow, it is the same in the lab and the comoving frame. In the comoving frame, the time for an Alfvén wave to communicate over this distance is $\tau_{\mathrm{A}}^{\prime}=$ $r \theta / v_{\mathrm{A}}^{\prime}$, in the lab frame $\tau_{\mathrm{A}}=\operatorname{\Gamma r} \theta / v_{\mathrm{A}}$. This is less than the time for the flow to reach a distance $r, t=r / \beta c \approx r / c$ if

$\theta<\frac{v_{\mathrm{A}}^{\prime}}{\Gamma c}=\frac{1}{\Gamma(1+1 / \xi)^{1 / 2}}$.

If the jet is wider than this, parts of the flow moving at angles separated by more than $\vartheta=(1+1 / \xi)^{-1 / 2} / \Gamma<1 / \Gamma$ have no time to communicate by an Alfvén wave in the time elapsed since the start of the flow, and behave as if they are causally disconnected from each other. Kink instability will thus be limited to an inner core of opening angle $\vartheta<1 / \Gamma$. In directions outside this angle, the instability is suppressed since it has to communicate from one side to then other across the axis of the jet. This reduces the fraction of the available magnetic energy that can be tapped by MHD processes.

On the other hand, magnetic energy release in the inner core so defined already starts very close to the source (where the length scale $\vartheta r$ is small). Thus only a fraction of the dissipation will happen in the optically thin, observable regions.

\subsection{General nonaxisymmetric outflows}

The conclusion from the above is that in the case of a perpendicular rotator, there is a well defined "magnetic dissipation radius" $r_{\mathrm{r}} \sim 10^{12} \mathrm{~cm}$ where most of the magnetic energy is dissipated. For a purely axisymmetric outflow along the rotation axis, on the other hand, only a fraction of the magnetic energy can be released, unless the opening angle is less than $\sim 1 / \Gamma$. This fraction probably dissipates close to the source, and not necessarily in the optically thin region where it could contribute to the observed emission. In intermediate cases, where both an axisymmetric and a nonaxisymmetric component are present, the magnetic field in the outflow changes direction on the length scale $L=\pi c / \Omega$, without completely changing direction. In such cases, the amount of the magnetic energy that can be released in directions outside the central core $\vartheta \sim 1 / \Gamma$ is of the order $B_{\mathrm{n}}^{2} / 8 \pi$, where $B_{\mathrm{n}}$ is the nonaxisymmetric part of the field. This is a significant fraction of the total magnetic energy unless the field is nearly axisymmetric. The axisymmetric jet case, though attractive as a computable model, is thus rather singular with respect to the question of magnetic dissipation which we address in this paper.

\section{Photospheric radius}

The reconnection radii derived above need to be compared with the radius $r_{\mathrm{p}}$ of photosphere in the outflow. If $r_{\mathrm{r}}$ is larger than $r_{\mathrm{p}}$, the dissipation of magnetic energy takes place mainly in the optically thin regime, and the dissipated energy is radiated away locally. If on the other hand $r_{\mathrm{r}}<r_{\mathrm{p}}$, the energy released from the initially ordered field configuration increases the internal energy of the plasma. The radial expansion of the flow converts this energy into kinetic energy of outflow. Though this may be useful in obtaining large Lorentz factors, it also implies that the magnetic energy left in the flow by the time it passes through 
the photosphere is small if $r_{\mathrm{r}} \ll r_{\mathrm{p}}$. The net output of a magnetically driven fireball with magnetic dissipation taking place mostly in the optically thick regime would be essentially the same as a standard non-magnetic fireball, with the attendant problem of a low efficiency of the production of radiation by internal shocks.

The photospheric radius in a steady relativistic outflow has been derived by Abramowicz et al. (1991). Assume a total amount of mass $M$ is ejected with a constant Lorentz factor $\Gamma$, at a rate $\dot{M}$ which is is constant in the time interval $0<t<\tau$, (and zero outside this interval). The radius at which the optical depth of the expanding shell reaches unity is then $r_{\mathrm{p}}=M \kappa /\left(8 \pi c \tau \Gamma^{2}\right)$, where $\kappa$ is the opacity. This a factor $2 \Gamma^{2}$ smaller than for photons propagating through a non-moving shell. This is a consequence of the expansion of the shell. The optical depth of a moving shell of constant (in time) density is a Lorentz invariant, hence the same for photons moving along the direction of the flow and those moving in the opposite direction (assuming also that the opacity is energy-independent). In a radial outflow, however, a photon sees a different history of mass density depending on its direction.

The result is widely used, e.g. Rees \& Mészáros (1994) and Beloborodov (2000), and is somewhat important for the quantitative estimates below. Since its correctness is sometimes questioned, we rederive it here in a different way from Abramowicz et al. (1991), by working in the lab frame. Let a photon be released at the inner edge $r_{\mathrm{i}}$ of the expanding shell at time $t=0$. With the aid of a Minkowski diagram, one finds that the photon exits at the outer edge of the shell at time $t_{\mathrm{e}}=\beta \tau /(1-\beta)$ and radius $r_{\mathrm{e}}=r_{\mathrm{i}}+d /(1-\beta)$ where $d=\beta c \tau$ is the shell thickness. To compute the optical depth traversed by the photon, we note that by the assumption of a constant outflow rate the density $\rho$ in the lab frame is only a function of $r$,

$\rho=\dot{M} /\left(4 \pi r^{2} \beta c\right)$

If the shell were static, the optical depth per unit length would just be $\kappa \rho$. Since the scattering medium is flowing outward at speed $v=\beta c$, the rate at which the photon overtakes its scattering centers (per unit length or time in the lab frame) is $1-\beta$ times the rate for a static shell (this is again easily seen in a Minkowski diagram). Summing up, the optical depth traversed by the photon is

$$
\begin{aligned}
\tau_{\mathrm{i}} & =\int_{r_{\mathrm{i}}}^{r_{\mathrm{i}}+d /(1-\beta)}(1-\beta) \kappa \rho \mathrm{d} r \\
& =\frac{M \kappa}{4 \pi r_{\mathrm{i}}} \frac{1}{r_{\mathrm{i}}+d /(1-\beta)} .
\end{aligned}
$$

For very small duration $\tau$, this reduces to the optical depth $M \kappa /\left(4 \pi r_{\mathrm{i}}^{2}\right)$ for a shell of mass $M$ and fixed radius $r_{\mathrm{i}}$.

The optical depth is a decreasing function of time as the shell expands and $r_{\mathrm{i}}$ increases. Define now the photospheric radius $r_{\mathrm{p}}$ as the value of $r_{\mathrm{i}}$ for which $\tau_{\mathrm{i}}=1$.
With $1 /(1-\beta) \approx 2 \Gamma^{2}$ and solving for $r_{\mathrm{i}}$ we get

$r_{\mathrm{i}}=c \tau \Gamma\left[\left(1+\frac{M \kappa}{4 \pi\left(c \tau \Gamma^{2}\right)^{2}}\right)^{1 / 2}-1\right]$,

The parameter

$$
\frac{M \kappa}{4 \pi\left(c \tau \Gamma^{2}\right)^{2}}=1.8210^{-5} \cdot \frac{E_{52}}{\xi+1}\left(\frac{\tau}{3 \mathrm{~s}}\right)^{-2}\left(\frac{\Gamma}{300}\right)^{-5}
$$

is small for relevant GRB parameters. Thus we find the photospheric radius to be

$r_{\mathrm{p}}=\frac{M \kappa}{8 \pi c \tau \Gamma^{2}}=\frac{E \kappa}{8 \pi c^{3} \tau \Gamma^{3}(\xi+1)}$

Numerically,

$r_{\mathrm{p}}=7.210^{10} \mathrm{~cm} \cdot(\xi+1)^{-1} E_{52}\left(\frac{\tau}{3 \mathrm{~s}}\right)^{-1}\left(\frac{\Gamma}{300}\right)^{-2}$.

As soon as the inner edge of the shell has expanded to this radius, radiation can escape from the entire shell.

\subsection{Pair opacity}

In addition to the baryons, pairs could also contribute to the opacity. The photospheric radius (68) then does not apply, because it includes only the constant opacity of scattering on the electrons associated with the baryonic mass. A general property of GRB fireball models is that the photosphere lies well outside the region where pairs contribute to the opacity. We summarize the argument here.

Assume a steady wind in which pairs dominate the kinetic energy flux and the opacity, and in which the kinetic energy of the pairs is a fraction $\xi_{ \pm}$of the total luminosity $E / \tau$. Let $T \approx 210^{8} \mathrm{~K}$ be the temperature at the pair photosphere (due to the steep dependence of the pair density on temperature, this value does not depend much on conditions in the outflow). Ignoring the opacity due to baryonic matter, the radius of the pair photosphere $r_{ \pm}$is (Usov 1994):

$$
\begin{aligned}
r_{ \pm}= & \left(\frac{\xi_{ \pm} E / \tau}{4 \pi \sigma_{\mathrm{SB}} T^{4} \Gamma^{2}}\right)^{1 / 2} \\
= & 210^{8} \mathrm{~cm} \cdot\left(\xi_{ \pm} E_{52}\right)^{1 / 2} \\
& \left(\frac{T}{210^{8} \mathrm{~K}}\right)^{-2}\left(\frac{\Gamma}{300}\right)^{-1}\left(\frac{\tau}{3 \mathrm{~s}}\right)^{-1 / 2}
\end{aligned}
$$

where $\sigma_{\mathrm{SB}}$ is the Stefan-Boltzmann constant and $\xi_{ \pm}$is the energy fraction in the pairs.

This value is much smaller than the photospheric radius (68). Thus, for typical baryon-loaded GRB parameters pairs annihilate before they reach the optically thin domain.

\section{Discussion}

Magnetic fields may well be the main agent tapping the rotational/gravitational energy in the central engines of 
GRB (for reviews see Mészáros 1999). Alternatives like the production of pair plasma fireballs by neutrino annihilation have turned out to have a rather small efficiency of conversion of gravitational energy (Ruffert et al. 1997). A fireball powered by magnetic fields ("magic hydrodynamics") can in principle produce $\gamma$-rays in the same way as in field-free mechanisms, namely by internal shocks in the optically thin part of the flow. A well-known problem with the internal shock mechanism, however, is the low efficiency of conversion to $\gamma$-rays Daigne \& Mochkovitch 1998), of the order of a few percent. This is hard to avoid, since efficient dissipation requires that a significant fraction of the energy in the outflow is in relative motion between parts moving at different velocities. The required large variations in $\Gamma$ lead to dissipation close to the source, however, in the optically thick regime. The dissipation taking place further out in the optically thin regime is due to smaller velocity differences that carry less energy. Ways to circumvent this limitation have been invented (Kumar \& Piran 2000; Beloborodov 2000; Lazzati et al. 1999). Even in these schemes, however, it seems unrealistic to expect the internal shocks to dissipate more than the external shock, hence the conflict between observed gamma-ray fluences and actual estimations of the energy dissipated by the external shock based on the observations of a few afterglows remains.

A magnetically powered outflow naturally carries a magnetic field with it ("Poynting flux"). This raises the question whether dissipation of this internal magnetic energy in the outflow can perhaps produce the observed radiation with a better efficiency.

We have addressed this question by considering a few possible scenarios for magnetic fields in GRB outflows. In the first, the "passive scenario", the magnetic field is assumed not to be responsible at all for powering the outflow, but only advected passively by an outflow produced by something else. We find that the maximum field strengths possible in this case are small compared with equipartition with the kinetic energy of the outflow, but still potentially significant for the effective production of synchrotron and/or inverse Compton emission in the internal shock model.

As magnetically-driven models we consider the case of a quasispherical outflow produced by a rotating nonaxisymmetric magnetic field, and the case of a jet-like outflow along the axis of a rotating axisymmetric field. The quasispherical case is like the models produced for the Crab pulsar (Coroniti 1990; Gallant \& Arons 1994). It has been developed for a completely baryon-free, pure pairplasma outflow by Usov (1994). The jet case is similar to magnetohydrodynamically driven wind models (Blandford \& Payne 1982; Sakurai 1985).

The magnetic field in all these cases is confined in an outward moving shell of width $c \tau$, where $\tau$ is the duration of the flow. If internal dissipation is ignored in the outflow, the total magnetic energy in the shell is constant, and the field strength $B$ varies with distance as $r^{-1}$ ( $B$ and $\tau$ measured in the observer frame). The configuration of the magnetic field in the shell is different in each case (see Figs. 1-3). If the central engine is time-dependent, for example in the form of a series of sub-bursts, magnetic shells like this are produced by each sub-burst, and the magnetic flux and energy in the shells is renewed with each burst (i.e. not limited by the magnetic flux of the central object).

We find that the MHD approximation (in the sense that the electric field in the fluid frame is small compared with the magnetic field) is safe out to large distances from the source, of the order $10^{19} \mathrm{~cm}$ or more. This is due to the relatively large amount of baryons in the outflow compared with, say, a pulsar wind situation. This simplifies the discussion of magnetic energy dissipation at least conceptually, since the energy release can be studied without detailed discussions of plasma processes (though they may enter again in the discussion of what produces the radiation).

The field strength in a magnetically driven outflow depends on the extent to which internal MHD processes have been able to dissipate magnetic free energy in the flow. In the absence of such processes, the magnetic energy density is typically of the same order as the kinetic energy density in magnetically driven flows, and the field is wellordered (large scale). In the internal shock model, such fields are sufficient to produce synchrotron and/or inverse Compton emission in the $\gamma$-ray range. The synchrotron case is favoured by a higher efficiency during the whole course of the burst. There are now some observational evidences that the magnetic field required for the afterglow emission represents a small fraction of the equipartition value whereas the prompt $\gamma$-ray emission via internal shocks is possible only with more intense fields close to the equipartition value (Galama et al. 1999). In the standard picture where the afterglow is due to the forward shock propagating in the external medium where no large scale field is present, it could mean that the generation of a local magnetic field behind the shock wave is inefficient. It is then possible that this locally generated field is also very small behind internal shocks within the shell but we show here that the large-scale field present in a magnetically driven outflow has no difficulty in most cases to supply the strength necessary for synchrotron and/or inverse Compton emission.

The rate of energy release through MHD processes like instabilities and fast reconnection is governed by the Alfvén speed. In the rest frame of the outflow, the Alfvén speed is of the order of the speed of light if the kinetic and magnetic energy fluxes are similar. In outflows produced by a perpendicular rotator, the magnetic field changes sign on the small length scale $c / \Omega$, and most of the magnetic energy can be dissipated by reconnection. The typical radius at which the energy release takes place in this case is $\sim 10^{12} \mathrm{~cm}$ for standard GRB parameters $\left(10^{52} \mathrm{erg}, 3 \mathrm{~s}\right.$, $\Gamma=300$ ). The photospheric radius, on the other hand, is small (mostly because of the relativistic effect discussed by Abramowicz et al. 1991), $\sim 10^{10} \mathrm{~cm}$. We can thus be 
confident that dissipation of energy stored in the magnetic field of the outflow occurs in the optically thin regime.

In the case of an outflow produced by a purely axisymmetric rotating magnetic object such changes of sign do not occur. Energy can still be released by kink instabilities, but for causality reasons these are not effective outside a narrow cone of angle $1 / \Gamma$ near the rotation axis (Sect. 5). The expected amount of magnetic dissipation in the optically thin regime is quite limited for such an axisymmetric field. A purely axisymmetric configuration, however, is a singular case, a priori unlikely for magnetic fields produced in a transient object like the central engine of a GRB. In the more likely case that a nonaxisymmetric field component is present as well, the energy in this component can be released by reconnection in nearly the same way as in the case of a perpendicular rotator.

Magnetic energy dissipation in the optically thin regime is probably not as simple as the shock dissipation in the internal shock model. In particular, the way in which nonthermal electron distributions are produced still needs to be investigated. It is likely that nonthermal radiation is again produced, as in the internal shock model, but the shape of the radiation spectrum may be more difficult to compute, as well as the typical time scale of the radiative process, which should be short compared to the expansion time scale (see $(46): t_{\mathrm{ex}}^{\prime} \sim 1 \mathrm{~s} \cdot(\Gamma / 300)^{-1}$ at $\left.10^{13} \mathrm{~cm}\right)$ to have a high radiative efficiency.

The main attraction of of GRB radiation produced by magnetic dissipation in a magnetically driven outflow is the efficiency with which the energy flux from the central engine can be converted into observable radiation. This efficiency is limited only by the ratio of Poynting flux to total energy flux in the flow. In the magnetically driven cases considered, this ratio can be close to unity. In the internal shock scenario, on the other hand, only a fraction $\lesssim 0.1$ of the energy is dissipated in the optically thin region. Since the bulk kinetic energy of the burst is dissipated in the afterglow, the internal shock model predicts the afterglows to dominate the energy output, which is probably inconsistent with current observations. In magnetic dissipation models such as those discussed here, the energy emitted in the afterglow can in principle be arbitrarily small compared with the prompt emission. It is not yet clear, however, how much of the magnetically dissipated energy can be in the form of $\gamma$-rays.

\section{References}

Abramowicz, M. A., Novikov, I. D., \& Paczyński, B. 1991, ApJ, 369,175

Baring, M. G. 1995, Ap\&SS, 231, 169

Bateman, G. 1980, MHD Instabilities (MIT Press, Cambridge) (Mass.), second edition

Begelman, M. C., \& Li, Z.-Y. 1992, ApJ, 397, 187

Begelman, M. C., \& Li, Z.-Y. 1994, ApJ, 426, 269

Beloborodov, A. M. 2000, ApJL, 539, L25

Berezinskii, V. S., \& Prilutskii, O. F. 1987, A\&A, 175, 309
Biskamp, D. 1993, Nonlinear magnetohydrodynamics, vol. 1 of Cambridge monographs on plasma physics, CUP, Cambridge (Mass.)

Bisnovatyi-Kogan, G. S., \& Ruzmaikin, A. A. 1976, Ap\&SS, 42,401

Blackman, E. G., \& Yi, I. 1998, ApJL, 498, L31

Blandford, R. D., \& Payne, D. G. 1982, MNRAS, 199, 883

Bykov, A. M., \& Mészáros, P. 1996, ApJL, 461, L37

Camenzind, M. 1987, A\&A, 184, 341

Coroniti, F. V. 1990, ApJ, 349, 538

Daigne, F., \& Mochkovitch, R. 1998, MNRAS, 296, 275

Daigne, F., \& Mochkovitch, R. 2000a, in preparation

Daigne, F., \& Mochkovitch, R. 2000b, A\&A, in press [astroph/0005193]

Daigne, F., \& Mochkovitch, R. 2000c, in 5th Huntsville Symposium on gamma-ray bursts, ed. M. Kippen, R. Mallozzi, \& G. Fishman, AIP

Galama, T. J., Briggs, M. S., Wijers, R. A. M., et al. 1999, Nature, 398, 394

Gallant, Y. A., \& Arons, J. 1994, ApJ, 435, 230

Galsgaard, K., \& Nordlund, Å. 1996, JGR, 101, 13445

Galsgaard, K., \& Nordlund, Å. 1997, JGR, 102, 231

Goodman, J. 1986, ApJL, 308, L47

Goodman, J., Dar, A., \& Nussinov, S. 1987, ApJL, 314, L7

Jackson, J. D. 1999, Classical electrodynmics (Wiley, New York), third edition

Katz, J. I. 1994, ApJL, 432, L107

Kennel, C. F., \& Coroniti, F. V. 1984, ApJ, 283, 694

Kluzniak, W., \& Ruderman, M. 1998, ApJL, 505, L113

Kobayashi, S., Piran, T., \& Sari, R. 1997, ApJ, 490, 92

Königl, A., \& Choudhuri, A. R. 1985, ApJ, 289, 173 (Erratum: ApJ, 305, 954)

Kumar, P., \& Piran, T. 2000 [astro-ph/9907096]

Lazzati, D., Ghisellini, G., \& Celotti, A. 1999, MNRAS, 309, L13

Lucek, S. G., \& Bell, A. R. 1996, MNRAS, 281, 245

Lucek, S. G., \& Bell, A. R. 1997, MNRAS, 290, 327

Medvedev, M. V., \& Loeb, A. 1999, ApJ, 526, 697

Melatos, A., \& Melrose, D. B. 1996, MNRAS, 279, 1168

Mészáros, P. 1999 [astro-ph/9912546]

Mészáros, P., Laguna, P., \& Rees, M. J. 1993, ApJ, 415, 181

Mészáros, P., \& Rees, M. J. 1992, MNRAS, 257, 29

Mészáros, P., \& Rees, M. J. 1993, ApJL, 418, L59

Mészáros, P., \& Rees, M. J. 1997, ApJL, 482, L29

Michel, F. C. 1969, ApJ, 158, 727

Narayan, R., Paczyński, B., \& Piran, T. 1992, ApJL, 395, L83

Paczyński, B. 1986, ApJL, 308, L43

Paczyński, B. 1998, ApJL, 494, L45

Panaitescu, A., \& Mészáros, P. 1999, ApJ, submitted

Papathanassiou, H., \& Mészáros, P. 1996, ApJL, 471, L91

Rees, M. J., \& Mészáros, P. 1994, ApJL, 430, L93

Rhoads, J. E., \& Paczynski, B. 1993, Am. Astron. Soci. Meeti., 183,0417

Ruffert, M., Janka, H.-T., Takahashi, K., \& Schäfer, G. 1997, A\&A, 319, 122

Sakurai, T. 1985, A\&A, 152, 121

Sari, R., \& Piran, T. 1997, MNRAS, 287, 110

Spruit, H. C. 1999, A\&A, 341, L1

Tavani, M. 1996, ApJ, 466, 768

Thompson, C. A. 1994, MNRAS, 270, 480

Thompson, C. A., \& Madau, P. 2000, ApJ, in press [astro-ph/9909111]

Usov, V. V. 1992, Nature, 357, 472

Usov, V. V. 1994, MNRAS, 267, 1035

Wijers, R. A. M. J., Rees, M. J., \& Mészáros, P. 1997, MNRAS, 288, L51

Woosley, S. E. 1993, ApJ, 405, 273 